JeFFrey Frederico LUI FILHO

Luiz Francisco Cintra BaCCARO2

TATIANE FERnANDES ${ }^{1}$

Délio Marques CONDE ${ }^{3}$

LÚCIA COSTA-PAIVA ${ }^{2}$

Aarão Mendes Pinto Neto ${ }^{2}$

\section{Artigo Original}

Palavras-chave

Menopausa/epidemiologia Climatério/fisiologia

Sintomas

Inquéritos demográficos

Keywords

Menopause/epidemiology

Climacteric/physiology

Symptoms

Population surveys

\title{
Epidemiologia da menopausa e dos sintomas climatéricos em mulheres de uma região metropolitana no sudeste do Brasil: inquérito populacional domiciliar
}

\author{
Factors associated with menopausal symptoms in women from a metropolitan \\ region in Southeastern Brazil: a population-based household survey
}

\section{Resumo}

OBJETIVOS: Avaliar a idade da menopausa e os fatores associados aos sintomas menopausais em mulheres de uma região metropolitana do sudeste do Brasil. MÉTODOS: Um estudo exploratório de corte-transversal foi realizado com 749 mulheres entre 45 e 60 anos (pesquisa de base populacional). A variável dependente foi a intensidade dos sintomas menopausais avaliada através do escore total do questionário Menopause Rating Scale (MRS). As variáveis independentes foram características sociodemográficas, problemas e hábitos de saúde, auto-percepção de saúde e antecedentes ginecológicos. A análise estatística foi realizada com o teste do $\chi^{2}$ e regressão de Poisson. RESULTADOS: A média etária das mulheres entrevistadas foi 52,5 ( $\pm 4,4)$ anos. Com relação ao estado menopausal, $16 \%$ das mulheres encontravam-se na pré-menopausa, o mesmo número na perimenopausa e $68 \%$ estavam na

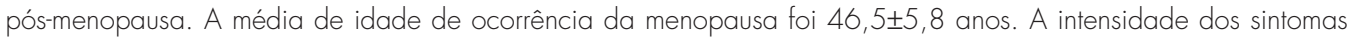
menopausais foi definida de acordo com a mediana do escore total do MRS e foi considerada severa para valores acima de 8. Depressão/ansiedade (RP=1,8; IC95\% 1,5-2,2; $\mathrm{P}<0,01)$, doenças osteoarticulares (RP=1,5; IC95\% $1,2-1,7 ; p<0,01)$, auto-percepção do estado geral de saúde regular, ruim ou péssimo ( $R P=1,4 ; 1 C 95 \%$ 1,2-1,7; $p<0,01)$, antecedente de algum aborto $(R P=1,3 ; \mid C 95 \% 1,1-1,5 ; p<0,01)$, tratamento para menopausa atual ou prévio (RP=1,2; IC95\% 1, 1-1,4; $p<0,01$ ), estar na perimenopausa ou pós-menopausa ( $R P=1,4 ; 1 C 95 \% 1,1-1,8$; $\mathrm{p}=0,01)$, número de partos normais $>1(\mathrm{RP}=1,2 ; \mathrm{IC} 95 \% 1,02-1,4 ; \mathrm{p}=0,02)$ e asma (RP=1,2; IC95\% 1,01-1,4; $\mathrm{p}=0,03$ ) se associaram a maior severidade de sintomas menopausais. Apresentar maior idade (RP=0,96; IC95\% 0,96-0,97; $p<0,01$ ) se associou a menor intensidade de sintomas da menopausa. CONCLUSÃO: A intensidade dos sintomas menopausais está relacionada a um amplo conjunto de fatores. Entender e controlar estes fatores pode auxiliar na redução dos sintomas menopausais, além de fornecer dados para definir grupos que necessitam maior atenção por parte dos serviços de saúde.

\section{Abstract}

PURPOSE: To determine the average age at the onset of menopause and to investigate menopausal symptoms in women in a metropolitan region in Southeastern Brazil. METHODS: A descriptive, exploratory, cross-sectional study was conducted with 749 women (a population-based household survey). The dependent variable was the intensity of menopausal symptoms assessed by th Menopause Rating Scale (MRS). The independent variables were sociodemographic data, health-related habits and problems, self-perception of health, and gynecological background. Statistical analysis was carried out by the $\chi^{2}$ test and Poisson regression using the backward selection criteria. RESULTS: The mean age of the women was $52.5( \pm 4.4)$ years. With regard to menopausal status, $16 \%$ were premenopausal, $16 \%$ perimenopausal and $68 \%$ postmenopausal. The mean age at the onset of menopause was $46.5( \pm 5.8)$ years. The intensity of menopausal symptoms was defined according to the median MRS score and was considered severe for values above 8. Depression/anxiety (PR=1.8; 95\% Cl 1.5-2.2; $\mathrm{p}<0.01$ ), rheumatic diseases (PR 1.5; 95\% Cl 1.2-1.7; $\mathrm{p}<0.011$, self-perception of health as fair/poor/very poor (PR 1.4; $95 \% \mathrm{Cl} 1.2-1.7 ; \mathrm{p}<0.01$ ), history of abortion (PR

\section{Correspondência \\ Aarõo Mendes Pinto Neto Rua Alexander Fleming, 101 - Cid. Univ. Zeferino Vaz \\ Barão Geraldo \\ CEP: 13083-881 \\ Campinas (SP), Brasil \\ Recebido \\ $26 / 01 / 2015$ \\ Aceito com modificacōes \\ $25 / 03 / 2015$}

Departamento de Tocoginecologia da Faculdade de Ciências Médicas da Universidade Estadual de Campinas - UNICAMP Campinas (SP), Brasil.

IPrograma de Pós-graduação, Faculdade de Ciências Médicas, Universidade Estadual de Campinas - UNICAMP - Campinas (SP), Brasil. 2Departamento de Tocoginecologia, Faculdade de Ciências Médicas, Universidade Estadual de Campinas - UNICAMP Campinas (SP), Brasil.

${ }^{3}$ Programa de Residência Médica de Mastologia, Hospital Materno Infantil de Goiânia - Goiânia (GO), Brasil.

Conflito de interesses: não há. 
1.3; $95 \% \mathrm{Cl} 1.1-1.4 ; \mathrm{p}<0.01$ ), current or previous treatment for menopausal symptoms (PR 1.2; $95 \% \mathrm{Cl} 1.1-1.4 ; \mathrm{p}<0.01$ ), peri- or postmenopausal status (PR 1.4; $95 \% \mathrm{Cl} 1.1-1.7 ; \mathrm{p}<0.01$ ), number of normal deliveries $>1$ (PR 1.2; 95\%Cl 1.02-1.4; $\mathrm{p}<0.01$ ) and asthma (PR 1.2; $95 \% \mathrm{Cl} 1.01-1.4 ; \mathrm{p}<0.01$ ) were associated with more severe menopausal symptoms. Older age (PR 0.96; 95\% Cl 0.96-0.97; $\mathrm{p}<0.01$ ) was associated with less severe symptoms. CONCLUSION: The severity of menopausal symptoms was related to a wild range of factors, especially presence of chronic diseases, a larger number of pregnancies, use of hormone therapy, and worse self-rated health. A better understanding of these factors can help to reduce the impact of symptoms on quality of life, and to identify groups of women who are likely to need more care during and beyond menopause.

\section{Introdução}

A menopausa é a interrupção permanente da menstruação. Pode ocorrer naturalmente ou de forma artificial, após procedimentos clínicos ou cirúrgicos que levem à parada da produção hormonal ovariana. Apesar de existir influência do eixo hipotálamo hipofisário, a menopausa natural é basicamente um evento ovariano, secundária à atresia fisiológica dos folículos primordiais, ocorrendo geralmente entre 40 e $55 \operatorname{anos}^{1}$. Dados recentes têm relacionado idade de ocorrência da menopausa e taxas de mortalidade ${ }^{2}$. Mulheres com menor idade à menopausa apresentam maior taxa de mortalidade por causas gerais e por algumas causas específicas como doenças cardiovasculares $^{3}$. Aquelas com menopausa em idade mais tardia apresentam maior taxa de mortalidade por neoplasias malignas ginecológicas hormônio-dependentes ${ }^{2}$. Além da influência direta da epidemiologia da menopausa sobre as taxas de mortalidade, o período climatérico costuma se apresentar com uma variedade de sintomas que afetam a qualidade de vida ${ }^{1,4}$. Dentre eles, destacam-se os vasomotores, como fogachos e sudorese, a atrofia genital e os transtornos psicológicos. Estes sintomas podem provocar prejuízo pessoal e implicação social de grande importância ${ }^{5-7}$.

Atualmente, existem poucos estudos de base populacional, nacionais ou latino-americanos, sobre a epidemiologia do climatério e da menopausa. A maioria dos dados disponíveis origina-se de países desenvolvidos da América do Norte e Europa. No Brasil, foi realizado um inquérito de base populacional com mulheres climatéricas na cidade de Campinas (SP) há mais de 15 anos $^{8-10}$. A escassez de trabalhos populacionais recentes demanda uma melhor avaliação das mulheres brasileiras. Com o objetivo de conhecer a média de idade da menopausa e os fatores associados à maior intensidade de sintomas climatéricos foi realizado estudo de base populacional na região metropolitana da cidade de Campinas, na região sudeste do Brasil.

\section{Métodos}

Foi conduzido um estudo descritivo exploratório, do tipo "corte transversal" entre setembro de 2012 e junho de 2013 com o objetivo de conhecer a média de idade da menopausa e os fatores associados à maior intensidade de sintomas climatéricos. Foram incluídas no estudo mulheres brasileiras natas com idade entre 45 e 60 anos, residentes na Região Metropolitana de Campinas. A incapacidade em responder a entrevista devido a qualquer motivo, como doenças e incompatibilidade de horários, foram considerados critérios de exclusão.

De acordo com o Instituto Brasileiro de Geografia e Estatística (IBGE), em 2010, a população da Região Metropolitana de Campinas era de 2.798.477 pessoas, sendo 1.423 .748 mulheres. Destas, 257.434 na faixa etária entre 45 e $60 \operatorname{anos}^{11}$. Para o cálculo do tamanho amostral considerou-se uma prevalência de mulheres com sintomatologia geral do climatério na cidade de Campinas (sintoma referência: fogacho) de $70 \%{ }^{10}$. Considerou-se uma diferença máxima desejada entre a proporção amostral e população de 3,5\%, um erro tipo I (alfa) de $5 \%$ e uma taxa de recusa de $20 \%$. Assim, estabeleceu-se um tamanho amostral de 820 mulheres a serem convidadas a participar do estudo ${ }^{12}$. A Região Metropolitana de Campinas (RMC) é composta por 19 municípios. Através de lista fornecida pelo IBGE, contendo os setores censitários numerados e claramente definidos, foram sorteados 92 setores, utilizando-se amostragem aleatória simples, equiprobabilística. Para o sorteio, foram excluídos os setores rurais, semirrurais e industriais. Foram listados os setores censitários urbanos contendo no mínimo 10 mulheres com idade entre 45 e 60 anos. Os setores em que não havia um mínimo de 10 mulheres nessa faixa etária foram agrupados com os setores vizinhos, de numeração posterior. Após o sorteio dos 92 setores, foi realizada seleção aleatória de duas quadras por setor e verificado o número de mulheres com idade entre 45 e 60 anos. Dessas, foram sorteadas 10 mulheres que foram entrevistadas. Auxiliares de pesquisa treinadas, guiadas por mapas e portando fichas de itinerário dirigiram-se até as residências e realizaram o convite para que as mulheres participassem do estudo. A entrevista pôde ser realizada no mesmo momento ou por telefone. Um total de 820 mulheres foram convidadas a participar do estudo, sendo que 71 recusaram-se. Com isso, a amostra final foi constituída por $749 \mathrm{mu}-$ lheres. Todas assinaram Termo de Consentimento Livre e Esclarecido previamente à realização da entrevista. 
O estudo foi aprovado pelo Comitê de Ética em Pesquisas do Departamento de Tocoginecologia, da Faculdade de Ciências Médicas da Universidade Estadual de Campinas (Unicamp), protocolo número 030/2011.

A variável dependente foi a intensidade dos sintomas da menopausa, dicotomizada em leve e intensa de acordo com a mediana do escore total do Menopause Rating Scale (MRS). O MRS é um questionário para avaliação dos sintomas da menopausa que já foi formalmente validado e traduzido para a Língua Portuguesa ${ }^{13}$, consistindo de onze questões abrangendo três domínios de sintomas: psicológico, somático e urogenital. Para cada questão, as mulheres podem escolher entre cinco possibilidades de resposta, graduadas de forma crescente quanto à intensidade dos sintomas. O escore total pode variar de zero (ausência de sintomas) a 44 (sintomatologia máxima). Este instrumento foi pré-testado pessoalmente ou por telefone em pacientes voluntárias do Ambulatório de Menopausa do Hospital da Mulher da Unicamp.

O questionário utilizado como instrumento para coleta dos dados foi estruturado em quatro seções: avaliação sociodemográfica, hábitos, problemas e autopercepção de saúde. Foi elaborado com base em um questionário brasileiro pré-existente (Critérios de Classificação Econômica do Brasil $)^{14}$. As variáveis independentes foram as características sociodemográficas: idade (anos); escolaridade (anos que frequentou instituições de ensino); estado marital (com ou sem companheiro/a); cor da pele (branca/não branca); realizar trabalho remunerado ( $\mathrm{sim} /$ não); renda familiar mensal (em reais); outras fontes de rendas ( $\operatorname{sim} /$ não); e estrato socioeconômico (A/B, C, $\mathrm{D} / \mathrm{E})$. Quanto aos hábitos e problemas de saúde consideramos: tabagismo (fumante atual, ex-fumante e não fumante); consumo de álcool (uma ou mais doses por semana, menos de uma dose por semana); prática de exercícios físicos $\geq 2$ vezes por semana ( $\operatorname{sim} /$ não). Para os problemas e percepção de saúde: estatura (metros); peso (quilogramas); índice de massa corporal $(<20$; 20-24,9; 25-29,9; >30); circunferência abdominal (centímetros); hipertensão arterial sistêmica ( $\operatorname{sim} /$ não); diabetes mellitus (sim/não); dislipidemia ( $\operatorname{sim} /$ não); infarto do miocárdio (sim/não); acidente vascular cerebral (sim/não); trombose venosa profunda ou embolia pulmonar (sim/não); osteoporose ou osteopenia ( $\operatorname{sim} /$ não); problemas osteoarticulares ( $\operatorname{sim} /$ não); asma ou bronquite (sim/não); tuberculose (sim/não); depressão, ansiedade ou outros transtornos psiquiátricos ( $\operatorname{sim} /$ não); câncer (sim/não). Nos antecedentes gineco-obstétricos analisamos: número de gestações $(0,1,2, \geq 3)$; número de partos normais $(0,1,2, \geq 3)$; número de cesáreas $(0,1$, $2, \geq 3)$; número de abortos $(0,1, \geq 2)$; número de filhos vivos $(0,1,2, \geq 3)$; número de parceiros sexuais durante toda a vida (até $1, \geq 2$ ); histerectomia prévia ( $\operatorname{sim} /$ não); ooforectomia bilateral ( $\operatorname{sim} /$ não); perineoplastia ( $\operatorname{sim} /$ não); estado menopausal (pré-menopausa se ciclos menstruais regulares; perimenopausa se ciclos menstruais irregulares ou amenorreia há menos de um ano; pós-menopausa se amenorreia há mais de um ano); e a autopercepção de saúde (ruim/péssima, regular, boa/excelente). A análise dos dados foi feita a partir da distribuição de frequências das características das mulheres estudadas. Em seguida, realizou-se análise bivariada para testar a associação entre a variável dependente (sintomas do climatério) e as variáveis independentes através do teste do $\chi^{2}$. Por fim, foi construído um modelo múltiplo de regressão de Poisson, para avaliar quais variáveis estavam independentemente associadas ao escore mais elevado do MRS. Para a regressão de Poisson, as razões de prevalência (RP) e respectivos IC95\% foram calculados para as variáveis significativas através do critério de seleção de variáveis backward. O nível de significância estatística foi estabelecido em $5 \%$ e o plano de amostragem por conglomerados (setores censitários) foi considerado nas análises bivariada e múltipla. Os softwares Statistical Package for the Social Sciences (SPSS) versão 20 e Stata versão 7 foram utilizados como ferramentas para a análise estatística.

\section{Resultados}

A avaliação foi realizada com 749 mulheres que responderam à entrevista. A média etária foi de 52,5 $( \pm 4,4)$ anos. Com relação ao estado menopausal, $16 \%$ encontravam-se na pré-menopausa, $16 \%$ na perimenopausa e $68 \%$ na pós-menopausa. A média etária de ocorrência da menopausa foi de 46,5 $( \pm 5,8)$ anos. As principais características clínicas e sociodemográficas (Tabela 1).

A variável dependente foi a intensidade dos sintomas do climatério. A média do escore total do MRS foi 9,8 $( \pm 7,9)$ e a mediana foi oito. Quanto às características sociodemográficas, a análise bivariada mostrou associação entre sintomas da menopausa e cor da pele não branca $(\mathrm{p}<0,01)$, sedentarismo $(\mathrm{p}<0,01)$, estar na pós menopausa $(\mathrm{p}<0,01)$, maior número de gravidezes $(\mathrm{p}=0,01)$, maior número de partos normais $(\mathrm{p}=0,04)$, maior número de abortos $(\mathrm{p}=0,01)$, maior número de parceiros sexuais durante a vida $(\mathrm{p}=0,01)$, idade na menopausa $\leq 48$ anos $(\mathrm{p}<0,01)$ e tempo desde a menopausa $>6$ anos $(\mathrm{p}=0,03)$ (Tabela 2). Quanto aos problemas de saúde, apresentar dislipidemia $(\mathrm{p}<0,01)$, osteoporose $(\mathrm{p}<0,01)$, problemas osteoarticulares $(\mathrm{p}<0,01)$, asma/bronquite $(\mathrm{p}=0,01)$, depressão/ansiedade $(\mathrm{p}<0,01)$, autopercepção do estado de saúde ruim 
ou péssimo $(\mathrm{p}<0,01)$, ter antecedente de histerectomia $(\mathrm{p}<0,01)$, ooforectomia bilateral $(\mathrm{p}<0,01)$ e perineoplastia $(\mathrm{p}<0,01)$ se associaram a maior intensidade de sintomas da menopausa na análise bivariada (Tabela 2).

A análise pela regressão múltipla de Poisson evidenciou associação entre intensidade dos sintomas climatéricos e apresentar depressão/ansiedade $(\mathrm{RP}=1,8$; IC95\% 1,5-2,2; $\mathrm{p}<0,01)$, doenças osteoarticulares $(\mathrm{RP}=1,5 ; \mathrm{IC} 95 \% 1,2-1,7 ; \mathrm{p}<0,01)$, autopercepção do estado geral de saúde regular, ruim ou péssimo ( $\mathrm{RP}=1,4 ; \mathrm{IC95 \%} \mathrm{1,2-1,7;} \mathrm{p}<0,01)$, antecedente de algum aborto ( $\mathrm{RP}=1,3$; IC95\% 1,1-1,5; $\mathrm{p}<0,01)$, tratamento para menopausa atual ou prévio $(\mathrm{RP}=1,2$; IC95\% 1,1-1,4; $\mathrm{p}<0,01)$, estar na perimenopausa ou pós-menopausa $(\mathrm{RP}=1,4$; IC95\% 1,1-1,8; $\mathrm{p}=0,01)$, número de partos normais $>1(\mathrm{RP}=1,2$; IC95\% 1,02-1,4; $\mathrm{p}=0,02)$ e asma $(\mathrm{RP}=1,2$; IC95\% 1,01-1,4; $\mathrm{p}=0,03)$. Apresentar maior idade (RP=0,96; IC95\% 0,96-0,97; $\mathrm{p}<0,01)$ foram associados à intensidade de sintomas da menopausa (Tabela 3).

Tabela 1. Características clínicas e sociodemográficas ( $n=749)$

\begin{tabular}{|c|c|c|}
\hline Característica & Total & $\%$ \\
\hline \multicolumn{3}{|l|}{ Idade (anos) } \\
\hline 45-49 & 228 & 30,4 \\
\hline $50-54$ & 242 & 32,3 \\
\hline $55-60$ & 279 & 37,2 \\
\hline \multicolumn{3}{|l|}{ Escolaridade (anos) } \\
\hline $1-4$ & 250 & 33,4 \\
\hline $5-8$ & 194 & 25,9 \\
\hline $9-11$ & 218 & 29,1 \\
\hline$\geq 12$ & 86 & 11,5 \\
\hline \multicolumn{3}{|c|}{ Realiza trabalho remunerado } \\
\hline Sim & 387 & 51,7 \\
\hline \multicolumn{3}{|l|}{ Renda familiar mensal } \\
\hline$<\mathrm{R} \$ 2.500,00$ & 304 & 47,3 \\
\hline \multicolumn{3}{|l|}{ Estado marital } \\
\hline Com companheiro & 552 & 73,7 \\
\hline \multicolumn{3}{|l|}{ Cor da pele } \\
\hline Branca & 514 & 68,6 \\
\hline Não branca & 235 & 31,4 \\
\hline \multicolumn{3}{|l|}{ Tabagismo } \\
\hline Tabagista & 125 & 16,7 \\
\hline Ex-Tabagista & 203 & 27,1 \\
\hline Não tabagista & 421 & 56,2 \\
\hline \multicolumn{3}{|c|}{ Consumo de álcool (1 ou mais dias/semana) } \\
\hline Sim & 93 & 12,4 \\
\hline \multicolumn{3}{|c|}{ Atividade física (2 ou mais vezes/semana) } \\
\hline Sim & 252 & 33,6 \\
\hline \multicolumn{3}{|l|}{ IMC } \\
\hline$<25$ & 235 & 33,8 \\
\hline$\geq 25$ & 461 & 66,2 \\
\hline
\end{tabular}

Tabela 2. Sintomas menopausais de acordo com características da população (apenas valores significativos - valores em porcentagens)

\begin{tabular}{|c|c|c|c|}
\hline \multirow{2}{*}{ Característica } & \multicolumn{2}{|c|}{ Valor do MRS } & \multirow{2}{*}{ Valor $\mathrm{p}^{3}$} \\
\hline & $\leq 8$ & $>8$ & \\
\hline \multicolumn{4}{|l|}{ Cor da pele } \\
\hline Não branca & 46,8 & 53,2 & $<0,01$ \\
\hline \multicolumn{4}{|c|}{ Atividade física (2 ou mais vezes/semana) } \\
\hline Não & 51,3 & 48,7 & $<0,01$ \\
\hline \multicolumn{4}{|l|}{ Dislipidemia } \\
\hline Sim & 43,6 & 56,4 & $<0,01$ \\
\hline \multicolumn{4}{|l|}{ Osteoporose } \\
\hline Sim & 36,4 & 63,6 & $<0,01$ \\
\hline \multicolumn{4}{|c|}{ Problemas osteoarticulares } \\
\hline Sim & 34,2 & 65,8 & $<0,01$ \\
\hline \multicolumn{4}{|l|}{ Asma ou bronquite } \\
\hline Sim & 41,0 & 59,0 & 0,01 \\
\hline \multicolumn{4}{|c|}{ Depressão ou Ansiedade } \\
\hline Sim & 34,2 & 65,8 & $<0,01$ \\
\hline \multicolumn{4}{|c|}{ Autopercepcão do estado de saúde } \\
\hline Ruim ou Péssima & 9,1 & 90,9 & $<0,01$ \\
\hline \multicolumn{4}{|l|}{ Estado menopausal } \\
\hline Pós-menopausa & 52,0 & 48,0 & $<0,01$ \\
\hline \multicolumn{4}{|c|}{ Número total de gestações } \\
\hline$\geq 3$ & 49,0 & 50,5 & 0,02 \\
\hline \multicolumn{4}{|c|}{ Número de partos normais } \\
\hline$\geq 3$ & 48,1 & 51,9 & 0,04 \\
\hline \multicolumn{4}{|l|}{ Número de abortos } \\
\hline 1 & 44,8 & 55,1 & 0,01 \\
\hline \multicolumn{4}{|c|}{ Número de parceiros durante toda a vida } \\
\hline$\geq 2$ & 49,3 & 50,7 & 0,01 \\
\hline \multicolumn{4}{|l|}{ Histerectomia prévia } \\
\hline Sim & 40,0 & 60,0 & $<0,01$ \\
\hline \multicolumn{4}{|c|}{ Ooforectomia bilateral prévia } \\
\hline Sim & 31,4 & 68,6 & $<0,01$ \\
\hline \multicolumn{4}{|l|}{ Perineoplastia prévia } \\
\hline $\operatorname{Sim}$ & 40,7 & 59,3 & $<0,01$ \\
\hline \multicolumn{4}{|l|}{ Idade na menopausa** } \\
\hline$\leq 48$ anos & 44,2 & 55,8 & $<0,01$ \\
\hline \multicolumn{4}{|c|}{ Tempo de menopausa*** } \\
\hline$>6$ anos & 47,9 & 52,1 & 0,04 \\
\hline
\end{tabular}

Tabela 3. Variáveis associadas aos valores totais de Menopause Rating Scale>8 (Análise múltipla por regressão de Poisson)

\begin{tabular}{lccc}
\hline Variável* & RP & $\begin{array}{r}\text { IC95\% } \\
\text { P/ RP }\end{array}$ & Valor p \\
\hline Idade (anos) & 0,96 & $0,96-0,97$ & $<0,01$ \\
Depressão ou ansiedade (Sim) & 1,84 & $1,51-2,24$ & $<0,01$ \\
Problemas ósteo-articulares (Sim) & 1,47 & $1,24-1,75$ & $<0,01$ \\
Estado geral da saúde (Regular, ruim, péssima) & 1,45 & $1,21-1,73$ & $<0,01$ \\
Número de abortos ( $\geq 1$ 1) & 1,27 & $1,10-1,47$ & $<0,01$ \\
Estado menopausal (Peri ou pós-menopausa) & 1,44 & $1,13-1,83$ & $<0,01$ \\
Asma ou bronquíte (Sim) & 1,21 & $1,02-1,45$ & 0,03 \\
Número de partos normais (>1) & 1,18 & $1,01-1,37$ & 0,04 \\
\hline *Análise considerando os setores censitários como unidades primárias de amostragem \\
\hline IC95\%: índice de confiança de 95\%; RP: razões de prevalência.
\end{tabular}




\section{Discussão}

O objetivo deste estudo foi o de conhecer a média de idade de ocorrência da menopausa e os fatores associados à maior intensidade dos sintomas climatéricos em mulheres com idade entre 45 e 60 anos residentes na região metropolitana de Campinas. A média etária de ocorrência do último sangramento menstrual foi $46,5 \pm 5,8$ anos. Este valor é baixo quando comparado aos relatados por estudos conduzidos em populações semelhantes. Em 2003, estudo com população residente na mesma região do país encontrou média de idade à menopausa de 51,2 anos $^{10}$. Em 2006, um grande estudo com mulheres latino-americanas saudáveis encontrou mediana de idade à menopausa de 48,6 anos ${ }^{15}$ e em 2008, estudo conduzido na região nordeste do Brasil obteve uma média de idade à menopausa de 48 anos $^{16}$. Quando a menopausa ocorre em uma idade inferior às médias relatadas, pode ser um sinal de envelhecimento prematuro ${ }^{17}$ e está associada a uma maior taxa de mortalidade de causa geral ${ }^{2,3,18}$. Estudo recente mostrou que mulheres com idade à menopausa inferior a 46,6 anos apresentam risco $16 \%$ maior de morte por qualquer causa ${ }^{2}$. Além disso, a mortalidade específica por causas coronarianas, respiratórias, geniturinárias e externas também pode ser maior nessas mulheres ${ }^{3}$. Uma possível explicação para maior mortalidade seria a deterioração precoce de células vasculares endoteliais, células de músculo liso e do miocárdio que apresentam receptores sensíveis à ação do estrogênio ${ }^{3,19}$.

O escore total médio do MRS foi 9,8 $( \pm 7,9)$. Os valores dos escores médios do questionário MRS podem ser muito discrepantes, variando de acordo com o país ou a cultura da população estudada. Algumas populações apresentam baixa intensidade de sintomas menopausais. Em 2007, estudo realizado no Equador relatou escore total médio do MRS de 6,4 $( \pm 3,6)^{20}$ e na Nigéria, em 2009, o valor total médio foi de $6,4( \pm 5,8)^{21}$. Outras populações, porém, apresentam escores do MRS bem mais elevados. Em 2008, estudo com mulheres chilenas encontrou valor total médio do MRS de 16,2 $\pm 8,5^{22}$. Recentemente, um estudo com mulheres turcas entre 40 e 65 anos, identificou média do escore total do MRS de 20,1 $( \pm 9,2)^{23}$. No Brasil, mulheres entre 35 e 65 anos residentes na região oriental amazônica apresentaram valor total médio do MRS de $15,6( \pm 8,8)^{24}$. No presente estudo, o escore total médio do MRS se assemelha ao encontrado em um grande estudo multicêntrico latino-americano que identificou valores médios de 11,3 ( $\pm 8,5$ pontos $)^{25}$. Possivelmente, a média etária relativamente alta das mulheres e o fato de uma grande parcela delas encontrarem-se na pós-menopausa $(68 \%)$ contribuiu para o escore baixo. Além disso, fatores socioeconômicos e educacionais, como menor renda familiar e menor escolaridade, podem ter influenciado a percepção dos sintomas menopausais e as queixas das mulheres climatéricas ${ }^{26}$.

A relação entre o estado menopausal e os sintomas climatéricos vem sendo investigada nos últimos anos. Em 2005, um grande estudo de coorte norte-americano ${ }^{27}$ identificou uma pequena incidência de fogachos na pré-menopausa, com aumento gradual dos sintomas vasomotores durante a perimenopausa precoce e um pico de incidência na perimenopausa tardia. Após a menopausa, a tendência é de que os fogachos tenham sua intensidade diminuída ${ }^{27}$. No presente estudo, a associação encontrada entre estado menopausal e intensidade dos sintomas climatéricos foi semelhante. Observou-se uma maior intensidade de sintomas nas mulheres na peri e pósmenopausa, porém sintomas menos intensos ocorreram em mulheres mais velhas. Em 2011, Blumel et al. ${ }^{28}$ investigaram a prevalência, a duração e o impacto dos sintomas menopausais na qualidade de vida de mulheres latino-americanas. Foi identificado um pico de sintomas vasomotores em mulheres na pós-menopausa precoce, com uma queda na prevalência durante a pós-menopausa tardia. Entretanto, mais da metade das mulheres ainda apresentavam sintomas vasomotores após 10 a 15 anos do último fluxo menstrual. Foi relatada também uma maior taxa de sintomas vasomotores em mulheres usuárias de terapia hormonal, quando o esperado seria uma relação inversa devido aos efeitos do estrogênio sobre os fogachos ${ }^{28}$. No presente estudo, também foi encontrada uma maior intensidade de sintomas vasomotores em mulheres usuárias de TH atualmente ou no passado. Possivelmente, ou a terapia hormonal utilizada não vem sendo eficaz, seja por dosagem inadequada seja por baixa adesão ao tratamento, ou as mulheres usuárias de $\mathrm{TH}$ experimentam sintomas menopausais de uma forma mais severa do que as não usuárias, independente do uso ou não de algum tipo de terapia.

A associação entre maior número de gestações e intensidade de sintomas climatéricos já foi relatada previamente. Mulheres chilenas com três filhos ou mais ${ }^{22}$ e nigerianas com maior número de gestações ${ }^{21}$ apresentaram maior intensidade de sintomas. Recentemente, estudo com mulheres equatorianas de meia-idade também relacionou maior número de gestações com maior intensidade de sintomas da menopausa ${ }^{29}$. Possivelmente, mulheres de maior paridade ainda são responsáveis pelo cuidado com crianças. Durante a transição menopausal, este tipo de responsabilidade pode ter uma influência negativa, levando a uma pior percepção dos sintomas ${ }^{30}$. No presente estudo, os sintomas também foram mais intensos em mulheres portadoras de doenças crônicas, como asma e doenças osteoarticulares, de maneira semelhante à encontrada em outras populações ${ }^{31,32}$. Acredita-se que devido ao maior acesso a serviços de saúde, as mulheres 
com doenças crônicas têm mais informação a respeito da menopausa e maior facilidade na identificação dos seus sintomas, o que pode justificar sua maior intensidade.

A transição menopausal, especialmente a perimenopausa precoce, é um período da vida que torna as mulheres mais propensas à irritabilidade, nervosismo e frequentes alterações no humor ${ }^{33}$. A presença de sintomas vasomotores, além de fatores, como encarar o envelhecimento, mudanças na composição corporal e a síndrome do ninho vazio, podem interferir negativamente ${ }^{34}$. Durante a perimenopausa e pós-menopausa precoce, as mulheres têm um risco de 2 a 4 vezes maior de apresentar um episódio depressivo superior $^{35}$. No presente estudo, observou-se associação entre maior intensidade de sintomas climatéricos e apresentar depressão/ansiedade, e também associação com autopercepção de estado de saúde ruim ou péssima. Essa relação pode ser causada tanto pelo efeito prejudicial de um pior estado psicológico sobre os sintomas da menopausa quanto pelo efeito negativo que os sintomas climatéricos trazem ao humor e à autopercepção de saúde individual.

No Brasil, há uma carência de dados epidemiológicos sobre menopausa e sintomas climatéricos. Acredita-se que a iniciativa de um estudo de base populacional sobre este tema é de grande importância para uma melhor compreensão de como as mulheres enfrentam essa fase da vida e quais são os fatores que se associam a uma pior percepção dos sintomas. Entretanto, este estudo apresenta limitações que não podem ser ignoradas. Como todo estudo de corte-transversal, não pode estabelecer relações de causa e efeito. Além disso, não há um ponto de corte pré-estabelecido no escore do MRS para classificação da intensidade dos sintomas menopausais. No presente estudo, como em muitos outros, essa classificação foi realizada através da mediana do escore total. As variáveis foram baseadas no autorrelato das pacientes e incertezas diagnósticas podem ter ocorrido, porém, como se trata de um estudo de base populacional, acredita-se que isto não invalida a importância dos resultados obtidos.

A idade de ocorrência da menopausa no presente estudo foi menor do que a relatada por estudos prévios. Este é um fato relevante em decorrência dos efeitos deletérios que uma privação estrogênica precoce pode causar. Novos estudos serão necessários para comprovar se há mesmo uma tendência de diminuição da idade de ocorrência do último sangramento menstrual nesta população. A intensidade dos sintomas climatéricos esteve relacionada a um amplo conjunto de fatores, destacando-se a presença de doenças crônicas, ter tido um maior número de gestações, uso de terapia hormonal e ter autopercepção de pior estado de saúde. Uma melhor compreensão desses fatores pode auxiliar na tentativa de reduzir o impacto dos sintomas na qualidade de vida, além de definir grupos de mulheres que necessitam maior atenção à saúde de forma multidisciplinar.

\section{Agradecimentos}

Este trabalho foi financiado pela Fundação de Amparo à Pesquisa do Estado de São Paulo (FAPESP), processo no 2011/14526-9.

\section{Referências}

1. Speroff L. The perimenopause: definitions, demography, and physiology. Obstet Gynecol Clin North Am. 2002;29(3):397-410.

2. Wu X, Cai H, Kallianpur A, Gao YT, Yang G, Chow WH, et al. Age at menarche and natural menopause and number of reproductive years in association with mortality: results from a median followup of 11.2 years among 31,955 naturally menopausal Chinese women. PLoS One. 2014;9(8):e103673.

3. Mondul AM, Rodriguez C, Jacobs EJ, Calle EE. Age at natural menopause and cause-specific mortality. Am J Epidemiol. 2005; 162(1 1):1089-97

4. Blumel JE, Castelo-Branco C, Binfa L, Gramegna G, Tacla X, Aracena $B$, et al. Quality of life after the menopause: a population study. Maturitas. 2000;34(1):17-23.

5. Woods NF, Mitchell ES. Symptoms during the perimenopause: prevalence, severity, trajectory, and significance in women's lives. Am J Med. 2005;118 Suppl 12B:14-24.

6. Waetjen LE, Ye J, Feng WY, Johnson WO, Greendale GA, Sampselle $C M$, et al. Association between menopausal transition stages and developing urinary incontinence. Obstet Gynecol. 2009; $114(5): 989-98$.

7. Freeman EW. Associations of depression with the transition to menopause. Menopause. 2010;17(4):823-7.

8. Pedro AO, Pinto-Neto AM, Costa-Paiva L, Osis M, Hardy E. [Climacteric women seeking medical care, Brazil]. Rev Saúde Pública. 2002;36(4):484-90. Portuguese.

9. Pinto Neto AM, Pedro AO, Hardy E, Osis M, Costa-Paiva LH, Martinez EZ. [Characterization of hormone replacement therapy users in Campinas, São Paulo]. Cad Saúde Pública. 2002;18(1):121-7. Portuguese.

10. Pedro AO, Pinto-Neto AM, Costa-Paiva LH, Osis M, Hardy EE. [Climacteric syndrome: a population-based study in Campinas, SP, Brazil]. Rev Saúde Pública. 2003;37(6):735-42. Portuguese.

11. Instituto Brasileiro de Geografia e Estatística (IBGE) [Internet]. Censo demográfico 2010. Rio de Janeiro: IBGE; 2010 [citado 2011 Mar]. Disponível em: <http://www.ibge.gov.br/home/ estatistica/populacao/censo2010/default.shtm> 
12. Kish L. Survey sampling. New York: John Wiley \& Sons; 1965.

13. Heinemann LA, Potthoff $P$, Schneider HP. International versions of the Menopause Rating Scale (MRS). Health Qual Life Outcomes. $2003 ; 1: 28$.

14. Associação Nacional de Empresas de Pesquisa (ABEP) [Internet]. Critério de Classificação Econômica Brasil. 2011 [citado 2011 Jun]. Disponível em: <http://www.abep.org/criterioBrasil.aspx>

15. Castelo-Branco C, Blumel JE, Chedraui P, Calle A, Bocanera R, Depiano $E$, et al. Age at menopause in Latin America. Menopause. 2006;13(4):706-12.

16. Malheiros ES, Chein MB, da Silva DS, Dias CL, Brito LG, Pinto-Neto AM, et al. [Climacteric syndrome in a Northeastern Brazilian city: a household survey]. Rev Bras Ginecol Obstet. 2014;36(4): 163-9. Portuguese.

17. Snowdon DA, Kane RL, Beeson WL, Burke GL, Sprafka JM, Potter $\mathrm{J}$, et al. Is early natural menopause a biologic marker of health and aging? Am J Public Health. 1989;79(6):709-14.

18. Jacobsen BK, Heuch I, Kvale G. Age at natural menopause and all-cause mortality: a 37-years follow-up of 19,731 Norwegian women. Am J Epidemiol. 2003;157(10):923-9.

19. Gruber CJ, Tschugguel W, Schneeberger C, Huber JC. Production and actions of estrogens. N Engl J Med. 2002;346(5):340-52.

20. Chedraui P, Aguirre W, Hidalgo L, Fayad L. Assessing menopausal symptoms among healthy middle aged women with the Menopause Rating Scale. Maturitas. 2007;57(3):271-8.

21. Olaolorun FM, Lawoyin TO. Experience of menopausal symptoms by women in a urban community in lbadan, Nigeria. Menopause. 2009; 16(4):822-30.

22. Prado $M$, Fuenzalida $A$, Jara $D$, Figueroa $R$, Flores $D$, Blumel JE. Evaluación de la calidad de vida en mujeres de 40 a 59 años mediante la escala MRS (Menopause Rating Scale). Rev Med Chile. 2008;136(12):1511-7.

23. Senturk Erenel A, Golbasi Z, Kavlak T, Dilbaz S. Relationship between menopausal symptoms and sexual dysfunction among married Turkish women in 40-65 age group. Int J Nurs Pract. 2014. [Epub ahead of print]

24. Silva AR, Tanaka ACA. Factors associated with menopausal symptoms severity in middle-aged Brazilian women from the Brazilian Western Amazon. Maturitas. 2013;76(1):64-9.
25. Chedraui $P$, Blümel JE, Baron $G$, Belzares $E$, Bencosme $A$ Calle $A$, et al. Impaired quality of life among middle aged women: a multicentre Latin American study. Maturitas. $2008 ; 61(4): 323-9$

26. Sievert LL, Anderson D, Melby MK, Obermeyer CM. Methods used in cross-cultural comparisons of somatic symptoms and their determinants. Maturitas. $2011 ; 70(2): 127-34$.

27. Avis NE, Brockwell S, Colvin A. A universal menopausal syndrome? Am J Med. 2005; 118 Suppl 12B:37-46.

28. Blumel JE, Chedraui P, Baron G, Belzares E, Bencosme A, Calle A, et al. A large multinational study of vasomotor symptom prevalence, duration, and impact on quality of life on middle-aged women. Menopause. $2011 ; 18(7): 778-85$.

29. Chedraui P, Pérez-López FR, Sánchez H, Sánchez P, Miranda $O$, Quispe $P$, et al. Application of the 10-item Cervantes Scale among mid-aged Ecuadorian women for the assessment of menopausal symptoms. Maturitas. 2014;79(1):100-5.

30. Chedraui P, Pérez-López F, Mendoza M, Morales B, Martinez MA, Salinas $A M$, et al. Severe menopausal symptoms in middle-aged women are associated to female and male factors. Arch Ginecol Obstet. 2010;281(5):879-85.

31. Waidyasekera H, Wijewardena K, Lindmark G, Naessen T. Menopausal symptoms and quality of life during the menopausal transition in Sri Lanka women. Menopause. 2009;16(1):164-70.

32. Metintas S, Arýkan I, Kalyoncu C, Ozalp S. Menopause Rating Scale as a screening tool in rural Turkey. Rural Remote Health. 2010; 10(1): 1230 .

33. Bromberger JT, Assmann SF, Avis NE, Schocken M, Kravitz HM, Cordal A. Persistent mood symptoms in a multiethnic community cohort of pre- and perimenopausal women. Am J Epidemiol. $2003 ; 158(4): 347-56$.

34. Hess R, Thurston RC, Hays RD, Chang CC, Dillon SN, Ness RB, et al. The impact of menopause on health-related quality of life: results from the STRIDE longitudinal study. Qual Life Res. 2012;21(3):535-44.

35. Bromberger JT, Kravitz HM, Chang YF, Cyranowski JM, Brown C, Matthews KA. Major depression during and after the menopausal transition: Study of Women's Health Across the Nation (SWAN). Psychol Med. $2011 ; 41$ (9):1879-88. 